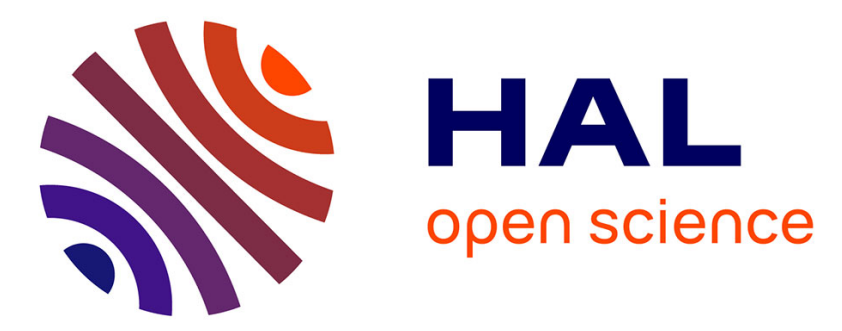

\title{
Force-Field Simulations of a Hydrated Lanreotide-Based Derivative: Hydration, Dynamics, and Numerical Evidence of Self-Assembly in Dimers
}

Florian Pinzan, Franck Artzner, Aziz Ghoufi

\section{- To cite this version:}

Florian Pinzan, Franck Artzner, Aziz Ghoufi. Force-Field Simulations of a Hydrated Lanreotide-Based Derivative: Hydration, Dynamics, and Numerical Evidence of Self-Assembly in Dimers. ACS Omega, 2020, 5 (39), pp.25423-25431. 10.1021/acsomega.0c03852 . hal-02992035

HAL Id: hal-02992035

https://hal-univ-rennes1.archives-ouvertes.fr/hal-02992035

Submitted on 12 Nov 2020

HAL is a multi-disciplinary open access archive for the deposit and dissemination of scientific research documents, whether they are published or not. The documents may come from teaching and research institutions in France or abroad, or from public or private research centers.
L'archive ouverte pluridisciplinaire HAL, est destinée au dépôt et à la diffusion de documents scientifiques de niveau recherche, publiés ou non, émanant des établissements d'enseignement et de recherche français ou étrangers, des laboratoires publics ou privés.

\section{(c)(1)}

Distributed under a Creative Commons Attribution| 4.0 International License 


\title{
Force-Field Simulations of a Hydrated Lanreotide-Based Derivative: Hydration, Dynamics, and Numerical Evidence of Self-Assembly in Dimers
}

\author{
Florian Pinzan, Franck Artzner, and Aziz Ghouf*
}

Cite This: ACS Omega 2020, 5, 25423-25431

Read Online

ACCESS | Lلll Metrics \& More | 国 Article Recommendations ｜ sl Supporting Information

ABSTRACT: Recently, self-organization of the cyclic octapeptide lanreotide and lanreotide-based derivatives in a nanotube to from a dimer structure has been experimentally evidenced. While the nature of the interactions between both monomers has been strongly investigated no molecular details of the hydration of the monomer and the formation of the dimer have been provided. Using molecular dynamics simulations, this work focuses on the structure, hydration, and dynamics of water and an analog of lanreotide. To do so, several models of monomers based on different schemes of partial charges and electrostatic interaction calculations are considered. By comparison with the experiments, we show that the model based on the combination of the AMBER force-field, CHELPG charge calculation, Ewald sum is the most relevant. Additionally, by mapping the interfacial hydration of the lanreotide monomer we evidence a heterogeneous surface in terms of hydrophilicity involving heterogeneous hydration. Furthermore, we show a slowdown in the translational dynamics of water molecules located close to the lanreotide surface. We also provide the molecular details of the self-assembly in the dimer in terms of structure, hydration, and energy.

\section{INTRODUCTION}

Self-organization is ruled by specific associations of molecules involving weak interactions such as hydrogen-bonding, hydrophobic, electrostatic, van der Waals, and $\pi-\pi$ interactions ${ }^{1-5}$ and provides different natural structures such as membranes, biological nanotubes, amyloid-beta protofibrils, or actin filaments. Nowadays, numerous applications such as drug delivery are based on the self-organization, ${ }^{1,6}$ and thus it is fundamental to well understand the physical processes ruling the self-assembly of these molecules. ${ }^{6,7}$ In the last few decades, many experimental and numerical works have been reported on the mechanism of self-assembly at the molecular scale and a connection between the geometrical complementarity, the chemical components and the weak interactions has been established. Peptides and lipids with self-assembling properties have been thus extensively explored given their simplicity in terms of shape and molecular interactions allowing the understanding of the molecular mechanisms driving the selfassembly processes. ${ }^{2,8-10}$

Among the materials which self-organize, the cyclic octapeptide lanreotide, a synthetic therapeutic peptide used mainly in the treatment of acromegaly, ${ }^{11-13}$ and shows a spectacular assembly because it self-associates into monodisperse nanotubes and coils in the presence of water, is less deeply studied."11 This packing exhibits as many as four hierarchical levels of organization, which, from the lowest to the highest, are $\operatorname{ref}^{7,11,12,14}$ (1) dimers of peptides essentially stabilized by hydrophobic effects and aromatic side chain interactions; (2) amyloid filaments generated by the packing of peptide dimers through an intermolecular antiparallel $\beta$-sheet network between the peptide backbones; (3) nanotubes generated by the lateral packing of 26 filaments, in which the $\mathrm{H}$-bond network lies flat on the nanotube surface; and (4) the packing of the nanotubes in a hexagonal lattice. The organizations in (2), (3), and (4) are clearly impacted by the formation of the dimers considered as the primitive brick that controls the highest packing from (2) to (4). Valéry et al., showed that three parameters are essential for the formation of lanreotide nanotubes: (i) the specificity of two of the three aromatic side chains, (ii) the spatial arrangement of the hydrophilic and hydrophobic residues, and (iii) the aromatic side chain in the $\beta$-turn of the molecule. ${ }^{14}$ The driving force of the hierarchical organization is the formation of the dimer structure controlled by the electrostatic and the van der Waals interactions between two monomers, and also by the hydration level of the monomer that should play an important role in dimer formation. A deep knowledge of the hydration of the monomer is therefore necessary to well understand and control the highest organization. Up to now, the experimental studies have been focused on the peptide-peptide interactions to elucidate the molecular mechanism controlling the selfassembly; however, the solvation/hydration state of the

Received: August 11, 2020

Accepted: September 10, 2020

Published: September 24, 2020 

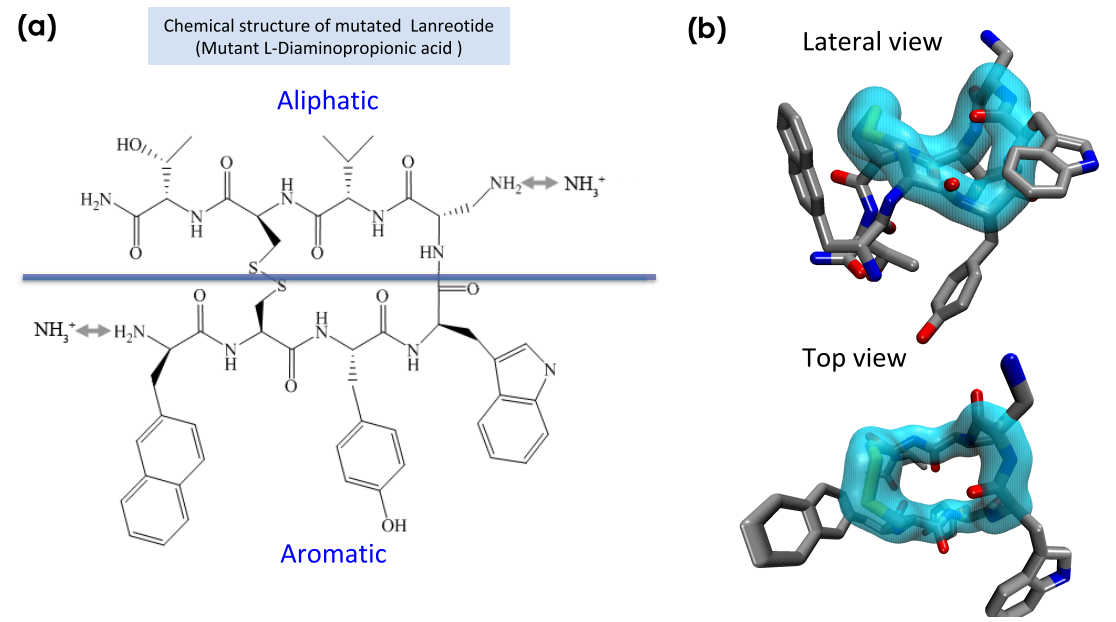

Figure 1. (a) Chemical structure of M-Lanr, highlighting the aliphatic and the aromatic sides, (b) lateral and top views of M-Lanr, from which hydrogen atoms have been removed. Gray, red, blue, and yellow correspond to carbon, oxygen, nitrogen, and sulfur atoms, respectively. Connolly's representation (cyan) has been used to highlight the M-Lanr backbone. The structure was obtained by molecular dynamics simulations at $300 \mathrm{~K}$ and 1 bar, wherein partial charges were calculated by the CHELPG scheme while the electrostatic interactions were evaluated using the Ewald sum.

monomer and the dimer of lanreotide and lanreotide-based derivatives was never explored.

Interestingly, it has been shown that the analog of lanreotide, mutant L-diaminopropionic acid (M-Lanr), built from eight connected ( $\mathrm{NH}_{2}$-(D)Naph-Cys-Tyr-(D)Trp-Lys-Val-Cys-Thr$\left.\mathrm{CONH}_{2}\right)$ residues, which self-organize into a dimer with similar unit cell parameters to lanreotide. ${ }^{15,16}$ According to Tarabout et al., the M-Lanr compound self-organizes into peptide coils in a crystal form. ${ }^{15,16}$ Contrary to lanreotide, the structure of M-Lanr and its unit cell parameters were then fully determined by X-ray diffraction (XRD), ${ }^{15,16}$ and this motivated our choice to consider M-Lanr in this study. As shown in Figure 1a, the analog of lanreotide is built from eight connected ( $\mathrm{NH}_{2}$-(D)Naph-Cys-Tyr-(D)Trp-Lys-Val-Cys-Thr$\mathrm{CONH}_{2}$ ) residues, producing an aliphatic and an aromatic backbone linked by a disulfur bridge leading to a cavity as highlighted in part (b) of Figure 1.

A relevant route to investigate the hydration state of M-Lanr and its ability to self-assemble into a dimer is probably the force-field simulations based on the atomistic description of matter. However, to the best of our knowledge, molecular dynamics simulation of lanreotide has never been performed, thus making it necessary to undertake the first work of modeling and validating the so-used force-field (set of empirical parameters allowing us to describe the interactions through a potential). This work aims to achieve this objective and to extract the most relevant atomistic force-field. While the AMBER force-field ${ }^{17}$ is considered to be robust to model proteins and macromolecules, several methods to calculate the partial charges to take into account the electrostatic interactions are used in the literature making a relevant choice difficult. On the one hand, this work is devoted to study the effect of charge and electrostatic interactions on the structure and hydration of M-Lanr, on the other hand, the structure, hydration, and dynamics of the monomer and the dimers are also studied.

\section{RESULTS AND DISCUSSION}

We report in Figure 2, the average of box length $(L)$ as a function of the electrostatic methodology. $L$ is connected to the density from $\rho=m / L^{3}$ where $m$ is the mass of the system.

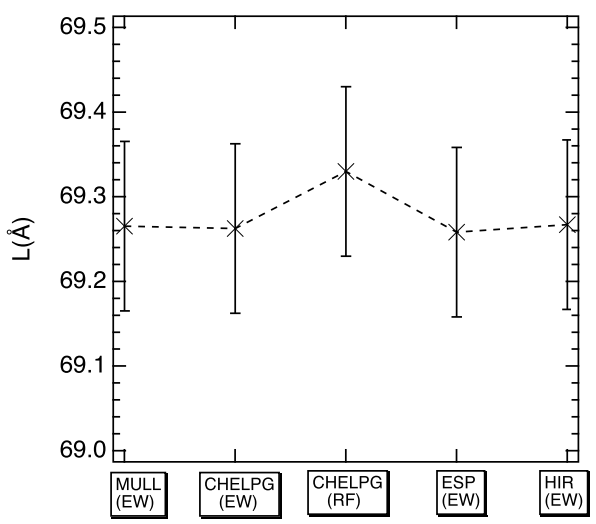

Figure 2. Box length as a function of the methodology of charge and the electrostatic interaction calculations.

As highlighted in Figure 2, all methods provide similar value of $L$ suggesting that the density is not method dependent. Microscopically, the impact of the electrostatic calculations on the distance between M-Lanr and the acetate ions was investigated. Indeed, it was established that the ionic systems show an ion pairing that plays an important role in the selfassembly process. ${ }^{18}$ We then report in Figure 3 the average distance between acetate ions and atoms of M-Lanr for all methods. The distance between M-Lanr and acetate ions have been calculated from the radial distribution function between the atoms of M-Lanr and the acetate ions. Indeed, the distance provided in Figure 3 corresponds to the first maximum in terms of probability of the radial distribution function. Although some differences are highlighted between all methods (charge calculations and both RF and EW methods), a distance greater than $12 \AA$ is observed, suggesting that acetate ions stay far from M-Lanr, indicating the absence of ion pairing. This is in good agreement with the osmometry measurements of the monomer. ${ }^{7}$ The large distance between the acetate ion and M-Lanr probably results in small impact of anions on the first and the second hydration shells of M-Lanr, suggesting that the ions do not participate in the self-assembly process from a hydration standpoint. This result was also obtained from a long simulation time of $150 \mathrm{~ns}$. We provided 
(a)

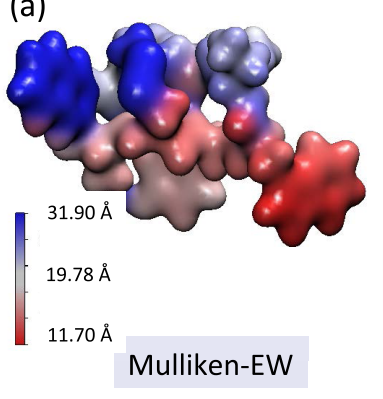

(d)

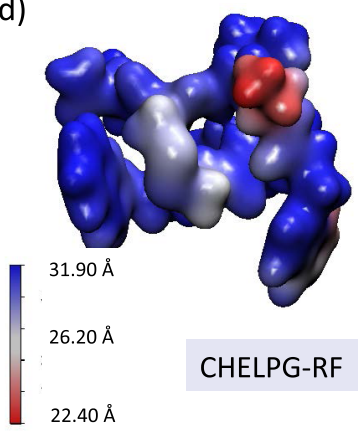

(b)

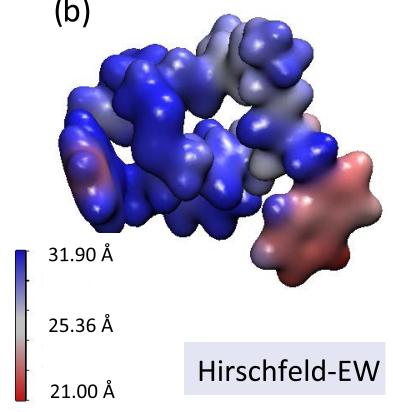

(e) (c)

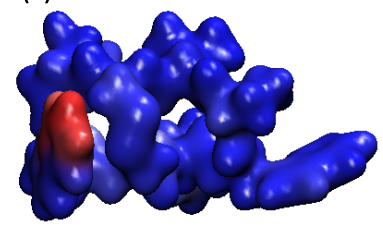

$31.90 \AA$

$29.58 \AA$

$27.26 \AA$

Figure 3. Three-dimensional representation of the average distance between the atoms of M-Lanr and the acetate ions for all methodologies (from (a) to (e)). Calculations were performed at $300 \mathrm{~K}$ and 1 bar by averaging on $3.5 \mathrm{~ns}$ the so-calculated distance between each atom of M-Lanr and anions. Part (f) represents a snapshot of M-Lanr to well identify the aromatic and the aliphatic backbones. Color scale represents the distance between each atom of M-Lanr and anions.

in Figure S1 of the Supporting Information, the time evolution of the distance between the center of mass of M-Lanr and acetate ions to confirm the time dependence during $150 \mathrm{~ns}$. Figure S1 shows that the distance between centers of mass of the acetate ions and M-Lanr in the CHELPG method during 150 ns exhibits the absence of ion pairing. The average distance between both centers of mass is also reported in Table 1 for all methods. Despite an error bar of around 7-8 Å Table

Table 1. Average Distance between the Acetate Ions and MLanr where the Error Bar is provided in Subscript ${ }^{a}$

$\begin{array}{lcccccc} & d_{\text {acetate-M-Lanr }} & \begin{array}{c}R_{\mathrm{g}} \\ (\AA)\end{array} & \begin{array}{l}R_{\mathrm{g} x} \\ (\AA)\end{array} & \begin{array}{l}R_{\mathrm{g} y} \\ (\AA)\end{array} & \begin{array}{l}R_{\mathrm{gz}} \\ (\AA)\end{array} & A \\ \text { MULLIKEN-EW } & 32.3_{7} & 5.4 & 3.1 & 3.2 & 3.2 & 0.06 \\ \begin{array}{l}\text { HIRSHFELD- } \\ \text { EW }\end{array} & 35.6_{8} & 5.6 & 3.2 & 3.4 & 3.2 & 0.15 \\ \text { CHELPG-EW } & 29.9_{9} & 5.7 & 3.4 & 3.0 & 3.2 & 0.21 \\ \text { CHELPG-RF } & 30.1_{8} & 5.6 & 3.1 & 3.2 & 3.5 & 0.17 \\ \text { ESP-EW } & 33.7_{6} & 5.7 & 3.8 & 2.8 & 3.1 & 0.15\end{array}$

${ }^{a}$ Gyration radius $\left(R_{\mathrm{g}}\right)$, its three components $R_{\mathrm{gx}}, R_{\mathrm{gy}}$, and $R_{\mathrm{gz}}$ and the asphericity $(A)$ of M-Lanr at $300 \mathrm{~K}$ and $1 \mathrm{bar}$ as a function of the computational methods.

1 shows the absence of ion pairing in all methods because of an average distance of $30 \AA$. The impact of the computational methods on the hydration level of M-Lanr was also investigated. We report in Figure 4 that the number of water molecules around each M-Lanr atom is at $5 \AA$. This distance corresponding to the first hydration shell was established from the calculation of the hydrogen bonds between water molecules and the M-Lanr atoms. Indeed, the distance between hydrogen atoms of a water molecule and a donor atom has to be smaller than $2.5 \AA$, according to the geometrical criteria established by Luzar and Chandler and based on the first principles calculations. ${ }^{19,20}$ To obtain a sufficient number of water molecules in the hydration shell, a distance of $5 \AA$ was considered. The length of a hydrogen bond is around $2.5 \AA$, whereas the radius of a water molecule is almost $2.7 \AA$, which corresponds to a hydration shell of $5 \AA$. As exhibited in Figure 4 the hydration number is found to be similar by all methods, which is in-line with the previous results on the acetate-MLanr distance. All methods provide similar hydrophobic and hydrophilic regions and highlight heterogeneous hydration and local clustering. Hydrophilic zones are located close to the aromatic side, whereas the aliphatic one is more hydrophobic. Interestingly, the backbone including the sulfur atoms lacks water molecules, defining a kind of a hydrophobic cavity. This peculiar hydration is the result of the fact that the polar groups are not accessible by the water molecules because the polar aliphatic moieties form a nonaccessible cavity with water molecules involving a confined region devoid of water molecules. On the contrary, aromatic zones are free and more accessible leading to an increase in water number in comparison with the aliphatic regions.

At this level of discussion, it seems that the methods for calculations of the electrostatic interactions and the partial charges slightly impact the hydration properties of M-Lanr. Regarding the internal structure of M-Lanr, the gyration radius and the asphericity were also calculated between all methods (see Table 1). The shape of the macrocyclic molecule was estimated from the inertia tensor $\mathbf{S}^{21}$ Diagonalization of $\mathbf{S}$ results in three eigenvalues, which sum the mean-squared radius of gyration $\left\langle R_{\mathrm{g}}^{2}\right\rangle$, the largest of which corresponds to an eigenvalue vector representing the long axis of the macrocycle. A useful descriptor of anisotropy is the asphericity $A$, which measures the deviation from a spherical form. ${ }^{21}$ If $A$ tends to 
(a)

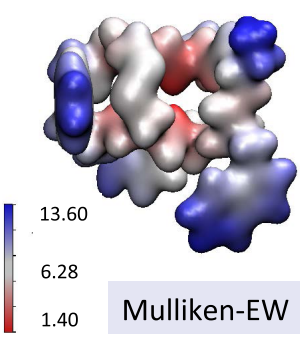

(d)

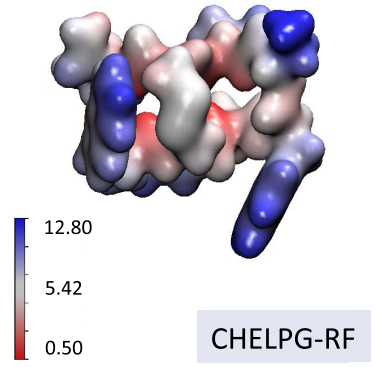

(b)

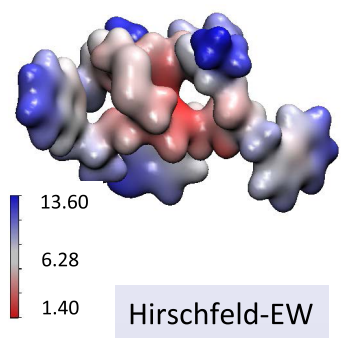

(e)

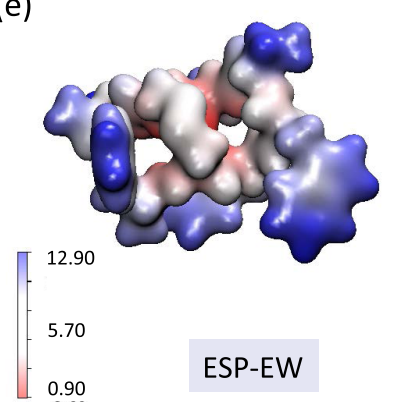

(c)

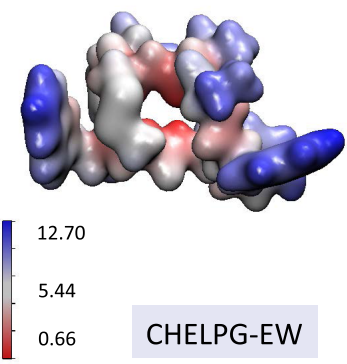

(f)

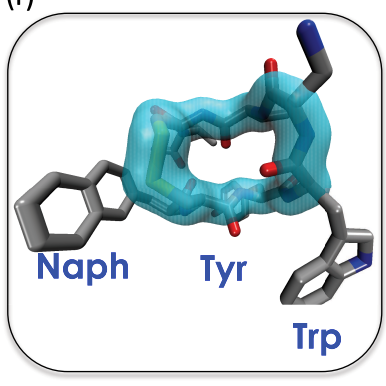

Figure 4. Three-dimensional representation of the average water number around the atoms of M-Lanr located at $5 \AA$ for all methodologies (from (a) to (e)). Calculations were performed at $300 \mathrm{~K}$ and 1 bar by averaging on $3.5 \mathrm{~ns}$, the so-calculated hydration number of each M-Lanr atom. Part (f) represents a snapshot of M-Lanr to well identify the aromatic and the aliphatic backbones. Color scale represents the number of water molecules located at $5 \AA$ around each M-Lanr atom.

zero, the macrocycle adopts a spherical conformation, while $A$ tends to 1 as the cycle becomes a thin rod. We report in Table $1, R_{\mathrm{g}}$ and its three components, $R_{\mathrm{g} x}, R_{\mathrm{g} y}$, and $R_{\mathrm{g} z}$, and $A$ as a function of the computational method. As shown in Table 1 , $R_{\mathrm{g}}$ seems to be slightly sensitive to the electrostatic interaction calculation. However, the components according to $x, y$, and $z$ directions are found to be different. Although the shape of $M$ Lanr is rather isotropic from the Mulliken and the Hirshfeld methodologies, anisotropy is found from the CHELPG and ESP calculations. As shown in Table 1, this difference is also recovered from the asphericity calculation. The structure of $\mathrm{M}$ Lanr is then impacted from the electrostatic interactions and partial charge calculations.

Translational dynamics of water was then evaluated from the calculation of the mean square displacement (MSD)

$$
\operatorname{MSD}(t)=\frac{\left\langle\sum_{t_{0}} \sum_{i=1}^{N}\left[\mathbf{r}_{\text {com }, i}\left(t+t_{0}\right)-\mathbf{r}_{\text {com }, i}\left(t_{0}\right)\right]^{2}\right\rangle}{N N_{0} t}
$$

where $\mathbf{r}_{\mathrm{com}, i}$ is the position of the center of mass of molecule $i$, $t_{0}$ is the time origin, $N$ is the number of molecules, and $N_{0}$ is the number of $t_{0}$. We report in Figure S2, the MSD of water molecules and we show that the water diffusion is unaffected by the method to handle the electrostatic interactions. Therefore, the hydration and the internal structure of $\mathrm{M}$ Lanr and the dynamics of water molecules do not allow us to make a decision on the most appropriated method. Furthermore, the MSD of water molecules in the first hydration was calculated and reported in Figure 5. As shown in Figure 5,dynamics of water molecules between 0 and $8 \AA$ around the M-Lanr is found to be smaller than the diffusion beyond $8 \AA$, corresponding to water bulk diffusion. As shown in Figure 4, close to the M-Lanr surface the water molecules

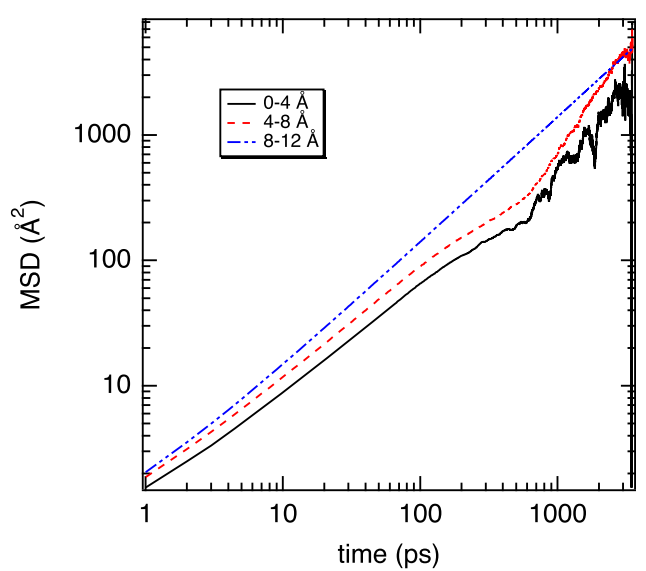

Figure 5. MSD of water molecules located in three shells around lanreotide $(0-4,4-8$, and $8-12 \AA)$ as a function of time.

are anchored by hydrogen bonds leading to a decrease in the translational degree of freedom.

The rotational dynamics has been evaluated from the calculation of the correlation function $(C(t))$ of the dipole moment vector $(\boldsymbol{\mu})$ of M-Lanr and water molecules. The dipole moment vector was calculated from $\boldsymbol{\mu}=\sum_{i=1}^{n} \sum_{j=1}^{m} q_{j}\left(\mathbf{r}_{j}\right.$ - $\left.\mathbf{r}_{\text {com, } i}\right)$ such that $n$ is the number of molecules, $m$ is the number of atoms of $i$ molecule, $q_{j}$ is the charge of atom $j, r_{j}$ is the position of atom $j$, and $r_{\mathrm{com}, i}$ is the position of the center of mass of molecule $i$. The correlation function of the dipole moment vector was then calculated from $C(t)=\left\langle\sum_{i=1}^{n} \boldsymbol{\mu}(t) \boldsymbol{\mu}(t\right.$ $=0)\rangle /\left\langle\sum_{i=1}^{n} \boldsymbol{\mu}(t=0) \boldsymbol{\mu}(t=0)\right\rangle$. We report in Figure 6a the $C(t)$ of M-Lanr as a function of time for all electrostatic calculations. As shown in Figure 6a, $C(t)$ strongly differs as a function of the computational methods. While the correlation function $C(t)$ obtained by the CHELPG-EW method shows a slow decay, 
(a)
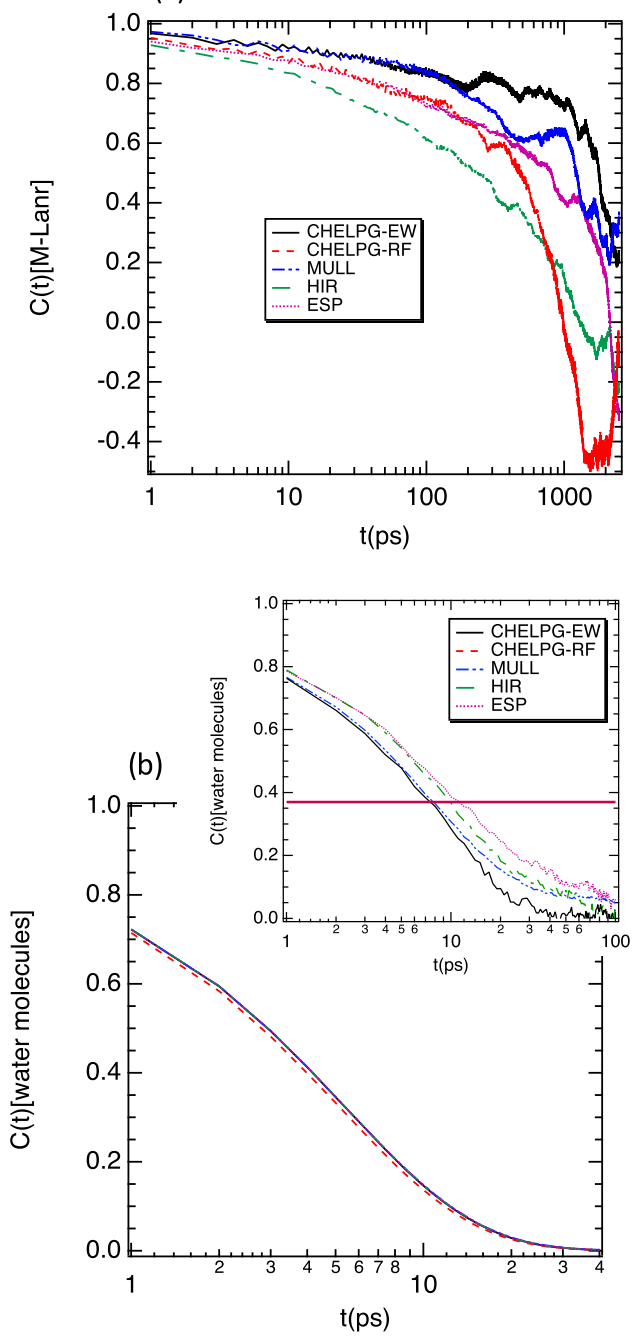

Figure 6. Correlation function $(C(t))$ of the dipole moment vector of M-Lanr (a) and water molecules (b) as a function of time and methods. In the inset of part (b) the correlation functions of water molecules located at the surface of M-Lanr (between 0 and $4 \AA$ ) as a function of time and methods. The horizontal line corresponds to the correlation function $C(t)=\exp ^{-1}$.

the HIR, MULL, CHELPG-RF, and ESP approaches present a fast decay. Figure 6a highlights that the different ability of partial charge calculation methods to handle the electrostatic interactions lead to different rotational dynamics, which could impact the self-organization of the monomer. Let us mention that a relaxation time makes sense if the correlation function of dipolar moments is decorrelated. In our case, the correlation function $(C(t))$ is decorrelated beyond $3 \mathrm{~ns}$ indicating that the system is dynamically relaxed and that the simulation time was sufficient to extract the rotational dynamics. Eventually, Figure $6 \mathrm{~b}$ exhibits that the rotational dynamics of water molecules is unchanged while the relaxation of M-Lanr is strongly influenced by the charge calculation. Interestingly, the relaxation time $\left(\tau_{\mathrm{R}}\right)$ can be evaluated by considering the Stokes-Einstein relation according to $1 / \tau_{\mathrm{R}}=k_{\mathrm{B}} T / 8 \pi \eta R^{3}$ where $k_{\mathrm{B}}$ is the Boltzmann's constant, $T$ is the temperature, $\eta$ is the viscosity of the solvent at $T$, and $R$ is the hydrodynamics radius of the solute. By considering a viscosity of $10^{-3} \mathrm{~Pa} \mathrm{~s}$ for water, a temperature of $298.15 \mathrm{~K}$ and a hydrodynamics radius of $R=7.3 \AA$ calculated from the diffusion coefficient $\left(3.0 \times 10^{-10} \mathrm{~m}^{2} / \mathrm{s}\right)^{7}$ as $D=k_{\mathrm{B}} T / 6 \pi \eta R, \tau_{\mathrm{R}}$ $=2.5 \mathrm{~ns}$ is in fair agreement with a few methods showing the relaxation time. Indeed, the following relaxation times were found, 2.2, 1.6, 1.5, 0.6, and 0.7 ns for CHELPG-EW, ESP MULL, HIRS, and CHELPG-RF methods, respectively. Relaxation time was calculated from the intersection between $C(t)$ and $\exp \left({ }^{-1}\right)$ that corresponds to the point where $C(t)$ is considered as decorrelated. These results shed light on the fact that the combination of the CHELPG approach and the Ewald sum provides a relaxation time of $2.2 \mathrm{~ns}$ that is in good agreement with the theoretical result of $2.5 \mathrm{~ns}$. Although the rotational dynamics of total water molecules was not impacted by the calculation of the partial charges and the electrostatic interactions, the rotational dynamics of water molecules located next to the surface of lanreotide is partially charge dependent. The inset in Figure $6 \mathrm{~b}$ shows different decay in $C(t)$ as a function of the charge calculations involving different relaxation times (time corresponding to the intersection between $C(t)$ and $\left.\exp \left({ }^{-1}\right)\right)$. This result highlights that the charge calculation on M-Lanr clearly impacts the local dynamics of water.

To ensure that the CHELPG and the Ewald sum are the best combination, we carried out molecular dynamics (MD) simulations of two monomers in aqueous solutions (two monomers with 5500 water molecules, in-line with the experiments ${ }^{7}$ ) to evaluate their ability to self-assemble into a dimer. ${ }^{7}$ We then performed MD simulations of two monomers in solution at $300 \mathrm{~K}$ and 1 bar by all methods. The reference distance between both monomers to obtain a dimer was determined by performing MD simulation of two monomers in the gas phase. This led to the formation of a dimer such that the average distance between both monomers is around $5.9 \AA$. In the aqueous phase and by considering the hydration of $\mathrm{M}$ Lanr, i.e., the diameter of one water molecule (3.7 $\AA$ ), we can assume that in solution the dimer is formed with the monomer-monomer distance between 6 and $10 \AA$. As shown Figure 7, the MULL and the ESP approaches failed

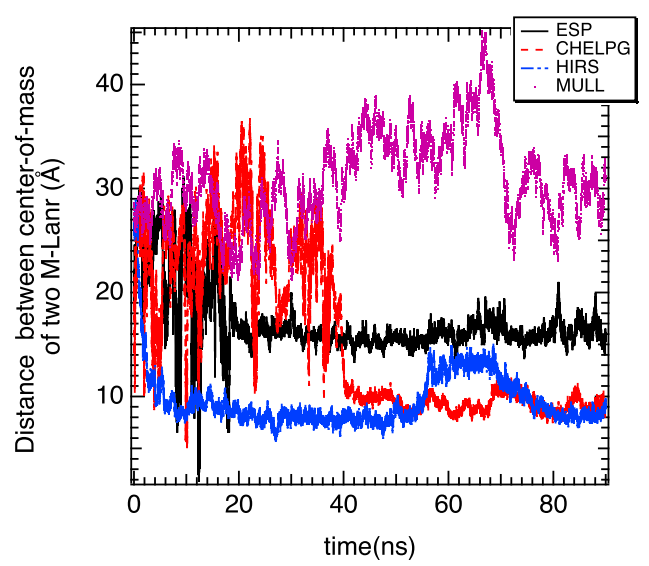

Figure 7. Distance between center of mass of two M-Lanr at $300 \mathrm{~K}$ for ESP, HIRS, and CHELPG methods using the Ewald sum.

to form the dimer even after $100 \mathrm{~ns}$, whereas the HIRS and the CHELPG methods succeeded in forming a stable dimer. Very interestingly, the combination of the reaction field approach and the CHELPG method did not result in the formation of the dimer; this shows the importance of handling the electrostatic interactions well. To confirm our results, two different simulations for each set of charges have been 


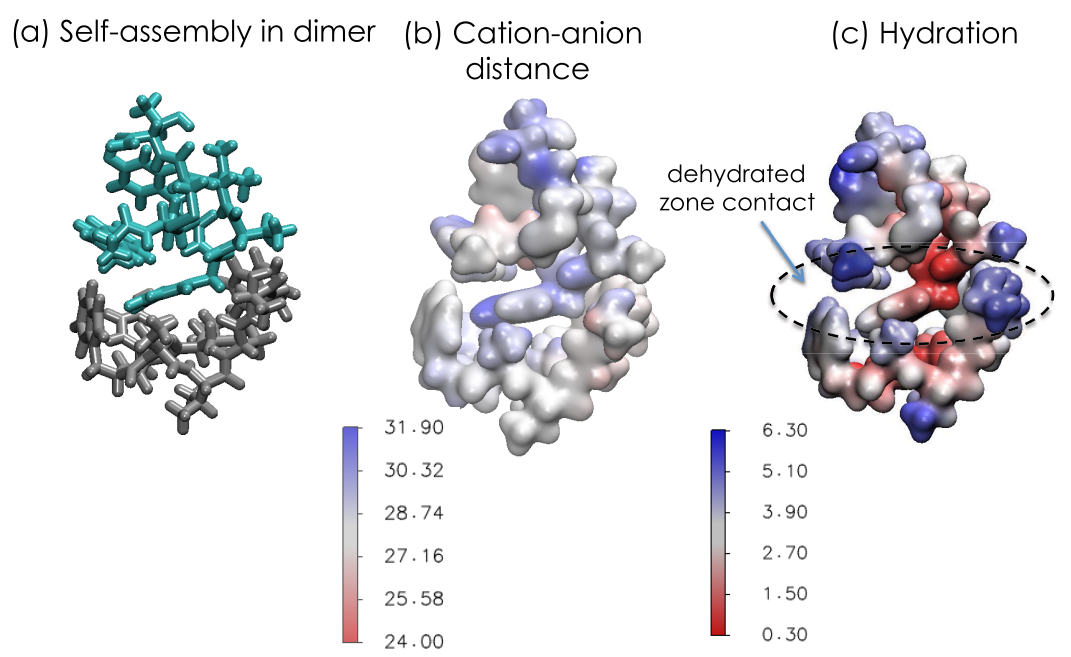

Figure 8. (a) Final configuration of the self-assembled dimer, where two monomers are represented in cyan and gray. (b) Three-dimensional representation of the average distance between the atoms of M-Lanr and the acetate ions. (c) Three-dimensional representation of the average water number around the atoms of M-Lanr located at $5 \AA$.

performed with different orientations and conformations of both monomers. Each configuration has been prepared by $\mathrm{MD}$ simulation of one monomer at $400 \mathrm{~K}$ to well sample the intramolecular degree of freedom and make sure that different conformations are obtained. In a second step, we built two configurations such that both monomers were inserted at (i) $30 \AA$ and (ii) $10 \AA$ separation, respectively. Results are presented in Table S1. As shown in Table S1, the dimers obtained by the CHELPG and the HIRS methods exhibited two different initial configurations.

Eventually, dynamical results and the ability to self-assemble seem to confirm that the combination of the CHELPG method to extract partial charges and the Ewald sum to handle electrostatic interactions are the most relevant to model hydrated M-Lanr. This good agreement with experiments (structure, diffusion, and self-assembly) shows that the so-used model is well-suited to describe the internal structure of lanreotide and the lanreotide-water interactions.

Interestingly, we report in Figure 8a the final configuration highlighting the self-assembly into a dimer. As shown in Figure $8 \mathrm{a}$, an inclusion complex seems to be formed, in which the tyrosine group of one monomer is included into the hydrophobic cavity, defined by the backbone containing the sulfur groups. Furthermore, the naphthalene and the tryptophan groups self-organize around the hydrophobic cavity to "capture" the tyrosine group. This organization is energetically favorable because the interactions between both monomers are maximized. Interestingly, Figure 8a shows that the dimer is ruled by the inclusion of the aromatic part in the hydrophobic cavity, which is in-line with the conclusions drawn by Valery et al. ${ }^{11}$ Although this conformation was stable in time because it was observed in $20 \mathrm{~ns}$, additional simulations are need to validate this stability. The fine study of the hydration and the structure of the dimer is out of the scope and will be presented in a future work focusing on simulations on the microscale. In part (b) of Figure 8, the threedimensional representation of the average distance between the atoms of M-Lanr and the acetate ions are reported. As shown in Figure $8 \mathrm{~b}$, the anions are found far from the dimer, suggesting the small role played by the acetate ions in the selfassembly process. However, Gobeaux et al. has shown that the counterions could control the size of lanreotide nanotube. ${ }^{22}$ In this work, we have evidenced that the counterions did not impact the formation of the dimer. However, these results are not in disagreement with the work of Gobeaux et al. because the formation of the nanotube involves thousands of dimers and then the so-generated electrostatic field could strongly impact the formation of the dimer. Therefore, our conclusions do not contradict the work of Gobeaux, which focused on the lanreotide nanotube, while our work is rather devoted to dimer formation.

Moreover, the three-dimensional mapping of the hydration state close to the M-Lanr surface is reported in Figure 8c. As evidenced in Figure 8c, the contact zone is depleted in water molecules in comparison with the hydration state of the monomer, indicating that strong dehydration of both monomers favors an entropic contribution to the self-assembly process for the dimer. Eventually, the binding energy between two monomers was calculated by considering both electrostatic (ELEC) and van der Waals (vdW) contributions. A binding energy of $-116.4 \mathrm{~kJ} / \mathrm{mol}( \pm 1.7 \mathrm{~kJ} / \mathrm{mol})$ was found, highlighting strong cohesion. Indeed, it was shown that below $-50.2 \mathrm{~kJ} / \mathrm{mol}$ the binding energy is considered as strong. ${ }^{23}$ The so-obtained dimer is then energetically stable suggesting that the dimer is the main brick in the self-assembly in the nanotube. Interestingly, the two ELEC and vdW contributions were also managed and a large difference was found. Indeed, the binding electrostatic energy is $-3.2 \mathrm{~kJ} / \mathrm{mol}$ while the vdW one is $-113.2 \mathrm{~kJ} / \mathrm{mol}$, suggesting that the dimer so-formed is a $\mathrm{vdW}$ complex and the driving force can be considered to be the $\mathrm{vdW}$ interactions rather than the electrostatic ones. Let us note that the uncertainty of the computed binding energy is evaluated by the block time average method on five blocks with 2500 configurations. $^{24}$

\section{CONCLUDING REMARKS}

In this work molecular dynamics simulations have been carried out to study the structure, hydration, and dynamics of an analog of lanreotide and its ability to self-assemble into a dimer. To do so, several models of monomers based on different schemes of partial charge and electrostatic interaction calculations have been considered.

We highlighted that the methods to calculate the partial charges and the electrostatic interactions were similar to 
describe the hydration while a few differences have been noted on the structure of M-Lanr. On the contrary, a difference in the rotational dynamics of M-Lanr and local water molecules between different methods was evidenced. Furthermore, a deep difference was found between RF and Ewald methods in terms of relaxation time, rotational dynamics, and self-assembly into a dimer. The comparison between the ESP and the CHELPG method evidenced a strong difference in structure and dynamics highlighting a difference between Dmol3 and Gaussian in favor of the latter. To make them equivalent, an additional study has to be undertaken on parameterization of Dmol3. We thus showed that the combination of the AMBER force-field, the CHELPG charge calculation method, and the Ewald sum, allowed us to obtain results in good agreement with the experiments, i.e., the observation of self-organization into a dimer and translational dynamics of M-Lanr.

By mapping the hydration at the surface of M-Lanr we have evidenced a hydrophobic cavity and surface involving heterogeneous hydration. Eventually, we showed that the stability of the dimer is ruled by the inclusion of the aromatic part in the hydrophobic cavity. Moreover, by calculating the different contributions of binding energy we established that the so-formed complex is controlled by van der Waals interactions rather the electrostatic ones.

\section{MODELS AND COMPUTATIONAL DETAILS}

4.1. Models. M-Lanr is positively charged (the total charge is $+2 \mathrm{e}$ ) and hence requires two additional anions to achieve electroneutrality. To be in-line with the experiments we opted for acetate ions as the counterion. ${ }^{7,11}$ From the experimental structure $^{15}$ obtained from XRD, hydrogen atoms have been added and geometry optimization was performed using a quantum scheme, in-line with the specific charge calculations detailed in Section 4.2. M-Lanr is built from 139 atoms and a $\mathrm{pdb}$ file is provided as the Supplementary Data (lanreotide.pdb). The AMBER ${ }^{17}$ force-field was used to model M-Lanr and the acetate ions. Indeed, the AMBER force-field is considered as relevant to simulate the organic and biological molecules. ${ }^{25}$ In a recent work, Man et al. have shown that the AMBER force-field could predict the structural properties of peptides in good agreement with the experiments. ${ }^{26}$ Fluitt and de Pablo also identified AMBERff99SB, AMBERff99SB*, and OPLS-AA force-fields to be the most suitable for studying the folding and aggregation of polypeptide. ${ }^{25}$ Water molecules were modeled by considering the rigid nonpolarizable TIP4P/ 2005 force-field. ${ }^{27}$ This water model was considered to be robust to quantitatively predict the physical properties of water, to account for the hydrogen-bonding network and to accurately model the hydration of ions and solutes. ${ }^{24,28-31}$ The force-field was divided in two contributions with respect to the intramolecular and the intermolecular interactions. Intramolecular contribution is based on stretching, angle, and dihedral potentials, while the intermolecular interactions were modeled from the electrostatic potential between partial charges and from the Lennard-Jones potential allowing us to take into account the van der Waals interactions. Mathematical expression of potentials is provided in the Supporting Information. Force-field parameters related to the equations given in the Supporting Information are provided in the files named parameters-CHELPG.txt, parameters-ESP.txt, parameters-HIR.txt, and parameters-ESP.txt. Initial configuration i.e., coordinates of M-Lanr and water molecules are also provided in the xyz.txt file.
Usually, partial charges of the AMBER force-field correspond to the averages obtained from quantum calculations based on reference molecules to obtain the "universal" parameters. Nowadays, computational resources allow us to carry out more accurate quantum calculations to extract more realistic charges. In this work, partial charges have been recalculated using DFT calculations, whereas the Lennard-Jones and the intramolecular AMBER parameters were conserved. Four methods have been compared, the CHELPG approach (CHarges from ELectrostatic Potentials using a grid-based method) ${ }^{32}$ from Gaussian ${ }^{33}$ software, the ESP (electrostatic potential fitting), ${ }^{34}$ the Hirshfeld ${ }^{35}$ and the Mulliken ${ }^{36}$ methods from the Dmol3 quantum package. ${ }^{37}$ These four methods were examined because they are considered as the most relevant to extract partial charges of nonperiodic systems. These approaches were based on a combination of the Becke exchange and Lee-Yang-Parr ${ }^{38}$ (B3LYP) correlation functional and all-electron core potentials. The B3LYP approach which belongs to the hybrid approximation of the exchangehybrid correlation functional is already very famous (porous material, small molecules, etc.). Let us note that similar results were obtained from the PBE method ${ }^{39}$ (generalized gradient approximation functional for exchange correlation energy) highlighting the reliability of the produced results. Additionally, the double- $\zeta$ numerical polarization (DNP) basis set was adopted to account for the d-type polarization in heavier atoms and p-type polarization in hydrogen atoms. This basis is similar to the $6-31 \mathrm{G}(\mathrm{d}, \mathrm{p})$ Gaussian-type basis set. ${ }^{40}$ Partial charges of acetate ions have been also calculated with the B3LYP method. Hirshfeld atomic populations are an alternative definition of atomic charges to the standard Mulliken (MULL) scheme providing a clear partitioning of the electron density. Let us note that the magnitude of the Hirshfeld (HIRS) charges is, in general, smaller than those for Mulliken, and the Hirshfeld charges are also less dependent on the basis set, as expected for a density-based population scheme. The CHELPG and the ESP method correspond to an atomic charge calculation scheme developed by Breneman and Wiberg, ${ }^{32,34}$ in which atomic charges are fitted to reproduce the molecular electrostatic potential at a number of points around the molecule.

4.2. Computational Details. The electrostatic interactions were truncated at $12 \AA$ and were calculated using both Ewald sum with a precision of $10^{-5}$ and the reaction field method that are detailed in the Supporting Information. Short range interactions were modeled by using the Lennard-Jones potential using a cutoff of $12 \AA$. Simulation boxes were cubic and periodic boundary conditions were applied in three directions. $\mathrm{MD}$ simulations were performed in the $N p T$ statistical ensemble such that, $N$ is the number of particles, $T$ is the temperature, and $p$ is the pressure. Molecular dynamics simulations were performed at $T=300 \mathrm{~K}$ and $p=1$ bar using a time step of $0.001 \mathrm{ps}$ to sample $10 \mathrm{~ns}$ (acquisition and equilibration phases). All MD simulations were carried out using the DL_POLY package, ${ }^{41}$ using a combination of velocity Verlet algorithm and the Nosé-Hoover thermostat and barostat algorithms. ${ }^{42,43}$ The initial configuration was built from a random distribution of water molecules in presence of M-Lanr and acetate ions. To be in-line with the experimental water fraction, ${ }^{7} 11000$ water molecules were considered in an initial cubic box length $(L)$ of $70 \AA$. All initial parameters have been provided in Table 2 . 
Table 2. Number of Water Molecules and Acetate Ions, Final Length of the Cubic Simulation Box, and Concentration in M-Lanr

\begin{tabular}{llccc} 
& $N_{\text {water }}$ & $L(\AA)$ & $N_{\text {acetate }}$ & concentration in M-Lanr $(\mathrm{mol} / \mathrm{L})$ \\
monomer & 11000 & 69.3 & 2 & $5 \times 10^{-3}$ \\
dimer & 5000 & 55.2 & 4 & $2 \times 10^{-3}$ \\
\hline
\end{tabular}

\section{ASSOCIATED CONTENT}

\section{s) Supporting Information}

The Supporting Information is available free of charge at https://pubs.acs.org/doi/10.1021/acsomega.0c03852.

Supplementary Data (ZIP)

Force-field equations, distances between both monomers (Table S1) and between anions and M-Lanr (Figure S1), and MSD of water molecules in all methods used for calculations of partial charges (Figure S2) (PDF)

\section{AUTHOR INFORMATION}

\section{Corresponding Author}

Aziz Ghoufi - Institut de Physique de Rennes, UMR 6251 CNRS, Université de Rennes 1, 35042 Rennes, France; ○ orcid.org/0000-0003-0877-7968; Email: aziz.ghoufi@ univ-rennes1.fr

\section{Authors}

Florian Pinzan - Institut de Physique de Rennes, UMR 6251 CNRS, Universite de Rennes 1, 35042 Rennes, France

Franck Artzner - Institut de Physique de Rennes, UMR 6251 CNRS, Université de Rennes 1, 35042 Rennes, France

Complete contact information is available at:

https://pubs.acs.org/10.1021/acsomega.0c03852

\section{Notes}

The authors declare no competing financial interest.

\section{ACKNOWLEDGMENTS}

A.G. is grateful to the CNRS for financial support through the project "PICS Waterloo".

\section{REFERENCES}

(1) Whitesides, G.; Mathias, J.; Seto, C. Molecular self-assembly and nanochemistry: a chemical strategy for the synthesis of nanostructures. Science 1991, 254, 1312.

(2) Caplan, M.; Moore, P.; Zhang, S.; Kamm, R.; Lauffenburger, D. Self-Assembly of a $\beta$-Sheet Protein Governed by Relief of Electrostatic Repulsion Relative to van der Waals Attraction. Biomacromolecules 2000, 1, 627.

(3) Ma, M.; Kuang, Y.; Gao, Y.; Zhang, Y.; Gao, P.; Xu, B. Aromatic-Aromatic Interactions Induce the Self-Assembly of Pentapeptidic Derivatives in Water To Form Nanofibers and Supramolecular Hydrogels. J. Am. Chem. Soc. 2010, 132, 2719.

(4) Chong, S. H.; Ham, S. Impact of chemical heterogeneity on protein self-assembly in water. Proc. Natl. Acad. Sci. U.S.A. 2012, 109, 7636.

(5) Mateescu, M.; Ispas-Szabo, P.; Assaad, E. Controlled Drug Delivery-The Role of Self-Assembling Multi-Task Excipients; Elsevier, 2015.

(6) Yoo, J.-W.; Irvine, D.; Discher, D.; Mitragotri, S. Bio-inspired, bioengineered and biomimetic drug delivery carriers. Nat. Rev. Drug Discovery 2011, 10, 521.

(7) Pouget, E.; Fay, N.; Dujardin, E.; Jamin, N.; Berthault, P.; Perrin, L.; Pandit, A.; Rose, T.; Valéry, C.; Thomas, D.; Paternostre, M.;
Artzner, F. Elucidation of the self-assembly pathway of lanreotide octapeptide into $\beta$-sheet nanotubes: role of two stable intermediates. J. Am. Chem. Soc. 2010, 132, 4230.

(8) Gazit, E. Self-assembled peptide nanostructures: the design of molecular building blocks and their technological utilization. Chem. Soc. Rev. 2007, 36, 1263.

(9) Heitz, F.; Morris, M. C.; Divita, G. Twenty years of cellpenetrating peptides: from molecular mechanisms to therapeutics. $\mathrm{Br}$. J. Pharm. 2009, 157, 195.

(10) Cui, H.; Webber, M.; Stupp, S. Self-assembly of peptide amphiphiles: From molecules to nanostructures to biomaterials. Pept. Sci. 2010, 94, 1.

(11) Valery, C.; Paternostre, M.; Robert, B.; Narayanan, T. G.-K. T.; Dedieu, J.; Keller, G.; Torres, M.; Cherif-Cheikh, R.; Calvo, P.; Artzner, F.; et al. Biomimetic organization: Octapeptide self-assembly into nanotubes of viral capsid-like dimension. Proc. Natl. Acad. Sci. U.S.A. 2003, 100, 10258.

(12) Valéry, C.; Artzner, F.; Paternostre, M. Peptide nanotubes: molecular organisations, self-assembly mechanisms and applications. Soft Matter 2011, 7, 9583.

(13) Hamley, I. Peptide Nanotubes. Angew. Chem., Int. Ed. 2014, 53, 6866.

(14) Valéry, C.; Pouget, E.; Pandit, A.; Verbavatz, J.; Bordes, L.; Boisdé, I.; Chrif-Cheikh, R.; Artzner, F.; Paternostre, M. Molecular origin of the self-assembly of Lanreotide into Nanotubes: A Mutational Approach. Biophys. J. 2008, 94, 1782.

(15) Tarabout, C. Autoassemblages Peptidiques d'analogues du Lanréotide: Contrôle du Diamètre des Nanotubes et Autres Morphologies. Ph.D. Thesis; Ecole Doctorale Sciences de la Matière, University Rennes 1, 2010.

(16) Cintrat, J.-C.; Ligeti, M.; Rousseau, B.; Artzner, F.; Tarabout, C.; Paternostre, M.-T.; Fay, N.; Cherif-Cheikh, R.; Valery, C. Novel Octapeptide Compounds and Therapeutic Use Thereof. U.S. Patent US20130252892A12011.

(17) Cornell, W.; Cieplak, P.; Bayly, C.; Gloud, I.; Merz, K.; Fergusson, D.; Spellmeyer, D.; Fox, T.; Caldwell, J.; Kollman, P. A Second Generation Force Field for the Simulation of Proteins, Nucleic Acids, and Organic Molecules. J. Am. Chem. Soc. 1995, 117, 5179.

(18) Rehm, T.; Schmuck, C. Ion-pair induced self-assembly in aqueous solvents. Chem. Soc. Rev. 2010, 39, 3597.

(19) Luzar, A.; Chandler, D. Effect of Environment on Hydrogen Bond Dynamics in Liquid Water. Phys. Rev. Lett. 1996, 76, No. 928.

(20) Ghoufi, A.; Artzner, F.; Malfreyt, P. Physical Properties and Hydrogen-Bonding Network of Water-Ethanol Mixtures from Molecular Dynamics Simulations. J. Phys. Chem. B 2016, 120, 793.

(21) Mendes, A.; Bonal, C.; Morel-Desrosier, N.; Morel, J.; Malfreyt, P. Molecular Dynamics Simulations of p-Sulfonatocalix[4]arene Complexes with Inorganic and Organic Cations in Water: A Structural and Thermodynamic Study. J. Phys. Chem. B 2002, 106, 4516.

(22) Gobeaux, F.; Fay, N.; Tarabout, C.; Meriadec, C.; Meneau, F.; Ligeti, M.; Buison, D.; Cintrat, J.-C.; Minh, K.; Nguyen, H.; Perrin, L.; Valéry, C.; Artzner, F. Structural role of counterions adsorbed on selfassembled peptide nanotubes. J. Am. Chem. Soc. 2012, 134, 723.

(23) Du, H.; et al. Principles of Inter-Amino-Acid Recognition Revealed by Binding Energies between Homogeneous Oligopeptides. ACS Cent. Sci. 2019, 5, 97.

(24) Ghoufi, A.; Malfreyt, P. Coarse Grained Simulations of the Electrolytes at the Water-Air Interface from Many Body Dissipative Particle Dynamics. J. Chem. Theory Comput. 2012, 8, 787.

(25) Fluitt, A.; de Pablo, J. An Analysis of Biomolecular Force Fields for Simulations of Polyglutamine in Solution. Biophys. J. 2015, 109, 1009.

(26) Man, V. H.; Derreumaux, P.; Ji, B.; Xie, X.-Q.; Nguyen, P. H.; Wang, J.; He, X. Effects of All-Atom Molecular Mechanics Force Fields on Amyloid Peptide Assembly: The Case of $\mathrm{A} \beta_{16-22}$ Dimer. J. Chem. Theory Comput. 2019, 15, 1440. 
(27) Abascal, J.; Vega, C. A general purpose model for the condensed phases of water: TIP4P/2005. J. Chem. Phys. 2005, 123, No. 234505.

(28) Zhu, H.; Ghoufi, A.; Szymczyk, A.; Belannec, B.; Morineau, D. Anomalous dielectric Behavior of Nanoconfined electrolytic solutions. Phys. Rev. Lett. 2012, 109, No. 107801.

(29) Renou, R.; Ghoufi, A.; Szymczyk, A.; Zhu, H.; Neyt, J.-C.; Malfreyt, P. Nanoconfined Electrolyte Solutions in Porous Hydrophilic Silica Membranes. J. Phys. Chem. C 2013, 117, 11017.

(30) Ding, M.; Szymczyk, A.; Goujon, F.; Soldera, A.; Ghoufi, A. Structure and dynamics of water confined in a polyamide reverseosmosis membrane: A molecular-simulation study. J. Membr. Sci. 2014, 458, 236.

(31) Ding, M.; Szymczyk, A.; Ghoufi, A. On the structure and rejection of ions by a polyamide membrane in pressure-driven molecular dynamics simulations. Desalination 2015, 368, 76.

(32) Breneman, C.; Wiberg, K. Determining atom-centered monopoles from molecular electrostatic potentials. The need for high sampling density in formamide conformational analysis. J. Comput. Chem. 1980, 11, 361.

(33) Frisch, M. J. et al. Gaussian 16, revision C.01; Gaussian Inc.: Wallingford, CT, 2016.

(34) Singh, U. C.; Kollman, P. An approach to computing electrostatic charges for molecules. J. Comput. Chem. 1984, 5, 129.

(35) Hirshfeld, F. Bonded-atom fragments for describing molecular charge densities. Theor. Chim. Acta 1977, 44, 129.

(36) Mulliken, R. Electronic Population Analysis on LCAO-MO Molecular Wave Functions. I. J. Chem. Phys. 1955, 23, 1833.

(37) Delley, B. An All-Electron Numerical Method for Solving the Local Density Functional for Polyatomic Molecules. J. Chem. Phys. 1990, 92, 508.

(38) Lee, C.; Yang, W.; Parr, R. G. Development of the ColleSalvetti correlation-energy formula into a functional of the electron density. Phys. Rev. B 1988, 37, No. 785.

(39) Perdew, J. P.; Burke, K.; Ernzerhof, M. Generalized Gradient Approximation Made Simple. Phys. Rev. Lett. 1996, 77, No. 3865.

(40) Petersson, G. A.; Al-Laham, M. A. A complete basis set model chemistry. II. Open-shell systems and the total energies of the firstrow atoms. J. Chem. Phys. 1991, 94, 6081.

(41) Todorov, I.; Smith, W.; Trachenko, K.; Dove, M. DLPOLY, CCP5 Program. J. Mater. Chem. 2006, 16, 1911.

(42) Nosé, S. A unified formulation of the constant temperature molecular dynamics methods. J. Chem. Phys. 1984, 81, No. 511.

(43) Hoover, W. G. Canonical dynamics: Equilibrium phase-space distributions. Phys. Rev. A 1985, 31, No. 1695. 